\title{
Atomic Physics Effects on Tokamak Edge Drift-Tearing Modes
}

\author{
T. S. Hahm \\ Princeton Plasma Physics Laboratory, Princeton University \\ Princeton, N.J.
}

\begin{abstract}
The effects of ionization and charge exchange on the linear stability of drift-tearing modes are analytically investigated. In particular, the linear instability threshold $\Delta^{\mathrm{Th}}$, produced by ion sound wave coupling [Phys. Rev. Lett. 40, 1500 (1978)] is modified. In the strongly collisional regime, the ionization breaks up the near cancellation of the perturbed electric, field and the pressure gradient along the magnetic field, and increases the threshold. In the semi-collisional regime, both ionization and charge exchange act as drag on the ion parallel velocity [Phys. Fluids B 4, 2567 (1992)], and consequently decrease the threshold by reducing the effectiveness of ion sound wave propagation.
\end{abstract}

PACS numbers: 52.35.Py, 52.30.Jb, 52.25.Gj, 52.55.Fa 


\section{Introduction}

The $\mathrm{L}$ to $\mathrm{H}$ transitions in tokamaks are usually accompanied by a change in magnetic activity near plasma boundary. 1,2 While most of the previous theoretical analyses ${ }^{3-11}$ of low mode number tearing instability are based upon tokamak parameters well inside the plasma boundary, the extreme edge plasma parameters are drastically different. For instance, in a diverted tokamak, the magnetic shear diverges to infinity as the last closed flux surface is approached from inside. This increased magnetic shear has a significant impact on various resistive MHD instabilities. ${ }^{12}$ It has been also speculated that the enhanced magnetic shear at the edge produced by current ramp down can make the $\mathrm{L}$ to $\mathrm{H}$ transition easier for Limiter configuration plasmas. ${ }^{13}$ Other edge specific phenomena are the atomic physics processes such as ionization and charge exchange. Recently, it has been shown that the ionization can influence the stability of edge dissipative drift wave significantly in both slab $14,1.5$ and toroidal ${ }^{16}$ geometries.

In this paper, we investigate the instability threshold condition of low mode number drift-tearing modes in the presence of ionization and charge exchange. Our theoretical model consists of Braginskii equations with the aforementioned atomic physics effects. Sheared slab geometry is considered for simplicity. We focus on the drift-tearing regime with $\omega_{* e} \gg \gamma_{\text {FKR }}$ (the classical tearing mode growth rate) $>\beta_{\mathrm{n}}$ (ionization rate), $\gamma_{\mathrm{v}}$ (charge exchange rate).

The principal results of this paper are as follows. The drift-tearing mode dispersion relations are derived analytically for both strongly collisional regime and semi-collisional regime. Sound wave dynamics, ionization and charge exchange effects are included in the analysis. In the strongly collisional regime, ionization reduces the effective force to drive the parallel current, and raises the instability threshold $\Delta^{\text {Th }}\left(\Delta^{\prime}>\Delta^{\text {Th }}\right.$ is required for instability) due to sound wave ${ }^{17}$ by $O\left(\beta_{n} \omega_{*} e^{2 / 3 / \gamma_{F K R}}{ }^{5 / 3}\right)$. This stabilizing influence persists when this 
threshold is exceeded, and the linear growth rate is reduced by the same order. The effect of charge exchange is small. In the semi-collisional regime, the threshold value of $\Delta^{\prime}$ for instability is reduced by $O\left(\left(\beta_{n}+\gamma_{v}\right) / \omega_{* e}\right)$. This destabilizing effect comes from the drag on the ion parallel motion due to ionization and charge exchange which reduces the ion sound wave effect.

The remainder of this paper is organized as follows. In Sec. II, the basic theoretical model is described, and the governing equations are presented. In Sec. III, the dispersion relations are derived in the absence of ion sound wave dynamics. The ion sound wave effects which produce the threshold are studied in Sec. IV. Finally, the implications of the principal results from the present studies are discussed in Sec.V.

\section{Theoretical Model}

The basic theoretical model for the collisional drift-tearing mode can be derived from the Braginskii fluid equations. We can include the atomic physics effects by taking moments of the Boltzmann equation. ${ }^{16}$ Considering a sheared slab system with an equilibrium magnetic field given by $B_{0}=B_{0}\left(z+y X / L_{s}\right)$, we have the following set of linearized cold ion fluid equations in terms of dimensionless variables.

$$
\begin{aligned}
& n_{e}=\left(\omega_{* e} / \omega\right) \Phi+\left(k_{11} C_{S} / \omega\right) V_{e}+\left(i \beta_{n} / \omega\right) n_{e} \\
& 0.51 v_{e i}\left(V_{i}-V_{e}\right)=i\left(v_{T e} 2 / C_{s}\right)\left\{k_{\|}\left(n_{e}-\Phi\right)+\left(\omega-\omega_{* e}\right) \Psi\right\}
\end{aligned}
$$




$$
\begin{aligned}
& \mathrm{n}_{\mathrm{i}}=\left(\omega_{* \mathrm{e}} / \omega+\rho_{\mathrm{s}}^{2} \nabla_{\perp}^{2}\right) \Phi+\left(\mathrm{k}_{\|} \mathrm{C}_{\mathrm{S}} / \omega\right) \mathrm{V}_{\mathrm{i}}+\left(\mathrm{i} \beta_{\mathrm{n}} / \omega\right) \mathrm{n}_{\mathrm{e}} \\
& \mathrm{V}_{\mathrm{i}}=\left(\mathrm{k}_{\|} \mathrm{C}_{\mathrm{S}} / \omega\right) \mathrm{n}_{\mathrm{i}}-\left(\mathrm{C}_{\mathrm{s}} \omega_{* \mathrm{e}} / \omega\right) \Psi-\left(\mathrm{i} \gamma_{\mathrm{v}} / \omega\right) \mathrm{V}_{\mathrm{i}}-\left(\mathrm{i} \beta_{\mathrm{n}} / \omega\right) \mathrm{V}_{\mathrm{i}} \\
& -\nabla_{\perp}{ }^{2} \Psi=\left(\mathrm{C}_{\mathrm{s}} \omega_{\mathrm{Le}} \mathrm{e}^{2 / \mathrm{v}_{\mathrm{Te}}}{ }^{2}\right)\left(\mathrm{V}_{\mathrm{i}}-\mathrm{V}_{\mathrm{e}}\right)
\end{aligned}
$$

In deriving Eqs (1)-(5), the electron temperature fluctuation and the gradient are ignored for simplicity. Thus, the radiative processes which couple only weakly to microtearing modes 19 through the thermal and pressure gradient along the magnetic field are also ignored. Here, Eqs. (1) and (2) are the density continuity equations for electrons and ions, respectively. The ionization $\beta_{n}=n_{N}\langle\sigma v\rangle_{\text {ion }}$ acts as a source in both continuity equations. Equation (3) is the Ohm's law. Equation (4) is the ion velocity equation with charge exchange $\gamma_{v}=n_{N}\langle\sigma v\rangle_{c x}$ and ionization acting as drag. The Ampere's law, Eq. (5), and the quasi-neutrality condition, $n_{e}=n_{i}$, close the system of equations. The normalization follows that of Ref. 16; i.e., $\mathrm{n}_{\mathrm{e}}=\mathrm{n}_{\mathrm{e} 1} / \mathrm{n}_{\mathrm{o}}, \mathrm{n}_{\mathrm{i}}=\mathrm{n}_{\mathrm{i} 1} / \mathrm{n}_{\mathrm{o}}, \mathrm{V}_{\mathrm{i}}=\mathrm{V}_{\mathrm{i} /} / \mathrm{C}_{\mathrm{s}}, \mathrm{V}_{\mathrm{e}}=\mathrm{V}_{\mathrm{e} /} / \mathrm{C}_{\mathrm{s}}, \Phi=\mathrm{e} \phi / \mathrm{T}_{\mathrm{e}}, \Psi=\mathrm{e} A_{\|} / \mathrm{T}_{\mathrm{e}}$ Other notations are standard; i.e., $v_{T_{e}}{ }^{2}=T_{e} / m_{e}, C_{s}{ }^{2}=T_{e} / M_{i}, \rho_{s}=C_{s} / \Omega_{c i}, \omega_{p e} e^{2}=$ $4 \pi n_{0} e^{2} / m_{e}, \omega_{* e}=k_{\vartheta} \rho_{s} C_{S} / L_{n}, L_{n}^{-1}=-d \ln n_{0} / d r$, and $k_{\|}=k_{\vartheta} X / L_{s}$.

We begin the analysis by rearranging Eqs. (1)-(5) to a form convenient for tearing mode analysis. Combining Eqs. (1)-(2), we get the vorticity equation

$\nabla_{\perp} 2 \Phi=\left(\mathrm{k}_{\| \mid} \mathrm{V}_{\mathrm{A}}^{2 / \omega)} \nabla_{\perp}^{2} \Psi\right.$

where $V_{A}=B_{0} /\left(4 \pi n_{0} M_{i}\right)^{1 / 2}$ is the Alfven velocity. The sound wave dynamics can be absorbed into Eq.(2) by eliminating $V_{i}$ from Eq. (4), 


$$
\left(1-i \beta_{n} / \omega\right) n_{e}-\left(\omega * e^{/ \omega)} \Phi=\rho_{s}^{2} \nabla_{\perp}^{2} \Phi-\left\{1+i\left(\gamma_{v}+\beta_{n}\right) / \omega\right\}^{-1}\left(k_{\|} C_{s}{ }^{2} / \omega^{2}\right)\left(k_{\|} n_{e}-\omega_{* e} \Psi\right)\right.
$$

$V_{i}-V_{e}$ can be eliminated from the Ohm's law by the use of Eq. (5),

$$
-\eta_{s} \nabla_{\perp}^{2} \Psi=i\left[k_{\|}\left(n_{e}-\Phi\right)+\left(\omega-\omega_{* e}\right) \Psi\right\}
$$

where $\eta_{\mathrm{s}}=0.51 v_{\mathrm{ei}} / \omega_{\mathrm{pe}}{ }^{2}$ is the Spitzer resistivity.

To facilitate the asymptotic analysis, we write Eqs. (6)-(8) in the ballooning space where the following quantities transform according to

$\rho_{s}^{2} \nabla_{\perp}^{2}=-b_{\vartheta}\left(1+S^{2} \vartheta^{2}\right)$

$\mathrm{k}_{\|}=-(\mathrm{i} / \mathrm{qR}) \partial_{\vartheta}$

with $b_{\vartheta}=k_{\vartheta}^{2} \rho_{s}^{2}$, and magnetic shear $S=$ dlnq/dlnr. After eliminating $\Psi$ and assuming $\mathrm{k}_{\mathrm{r}}{ }^{2} \gg \mathrm{k}_{\vartheta}{ }^{2}$ ( fast radial variation), we have

$$
\begin{aligned}
& \left(\mathrm{V}_{\mathrm{A}} / \mathrm{qR}\right)^{2} \partial_{\vartheta} \vartheta^{2}\left\{1+i \eta\left(\omega-\omega_{* e}\right)^{-1} \vartheta^{2}\right\}^{-1} \partial_{\vartheta}\left(\mathrm{n}_{\mathrm{e}}-\Phi\right)-\omega\left(\omega-\omega_{* e}\right) \vartheta^{2} \Phi=0 \\
& \left(\mathrm{C}_{\mathrm{S}} / \mathrm{qR}\right)^{2} \partial_{\vartheta}\left\{1+i \eta\left(\omega-\omega_{* e}\right)^{-1} \vartheta^{2}\right\}^{-1} \partial_{\vartheta}\left\{\left(1+i \eta \omega^{-1} \vartheta^{2}\right) \mathrm{n}_{\mathrm{e}}-\left(\omega_{* \mathrm{e}} / \omega\right) \Phi\right\} \\
& =-\left\{1+\mathrm{i}\left(\gamma_{\mathrm{v}}+\beta_{\mathrm{n}}\right) / \omega\right\} \omega\left(\omega-\omega_{* \mathrm{e}}\right)\left\{\left(1-i \beta_{\mathrm{n}} / \omega\right) \mathrm{n}_{\mathrm{e}}-\left(\omega_{* \mathrm{e}} / \omega-\mathrm{b}_{\vartheta} \mathrm{S}^{2} \vartheta^{2}\right) \Phi\right\}
\end{aligned}
$$

where $\eta=\eta_{s} k_{\vartheta}{ }^{2} S^{2}$. Equation (11) is the vorticity equation with the first term representing the 
field line bending and the second term representing the ion inertia. Equation (12) is the density evolution equation with the first term describing the parallel compression due to ion sound wave dynamics and the terms in the last bracket $\{\ldots\}$ representing the time evolution of density, the ionization source, $\mathbf{E} \times \mathbf{B}$ advection of the mean density, and the polarization drift in the order of their appearance.

\section{Lowest Order Dispersion Relation}

In this section, we derive the dispersion relations for both strongly collisional drift-tearing mode and semi-collisional drift-tearing mode in the absence of the ion sound wave dynamics for simplicity. We also note that the charge exchange effects do not enter in the absence of ion sound wave. However, ionization modifies the dispersion relations.

In the ideal MHD region where resistive effects are negligible, Eqs. (11) and (12) become,

$\partial_{\vartheta} \vartheta 2 \partial_{\vartheta}\left(n_{c}-\Phi\right)=0$

$\partial_{\vartheta}\left\{n_{\mathrm{e}}-\left(\omega_{* \mathrm{e}} / \omega\right) \Phi\right\}=0$

The large- $\vartheta$ asymptotic behavior of the solution is

$\Phi=\left(\omega_{* \mathrm{e}} / \omega\right) \mathrm{n}_{\mathrm{e}} \approx 1+\mathrm{r}_{0} \Delta^{\prime} / 2 \pi^{2} \mathrm{NqS} \vartheta$

where $\Delta^{\prime}$ is the standard tearing stability parameter quantifying the magnetic free energy 
available from the equilibrium current density gradient, $\Delta^{\prime}=\operatorname{dln} \Psi /\left.\mathrm{dr}\right|_{+0}-\mathrm{dln} \Psi /\left.\mathrm{dr}\right|_{-0}$. Here, $r_{0}$ is the minor-radial location of the rational surface, $\mathrm{N}$ is the toroidal mode number of the perturbation.

In the intermediate region, the resistive diffusion rate across the magnetic field line is comparable to the mode frequency $\left(\eta \vartheta^{2} \approx \omega-\omega_{* e}\right)$, and the inertial effects are ignorable. Then, Eqs. (11) and (12) simplify to

$\partial_{\vartheta} \vartheta^{2}\left\{1+i \eta\left(\omega-\omega_{* e}\right)^{-1} \vartheta^{2}\right\}^{-1} \partial_{\vartheta}\left(n_{e}-\Phi\right)=0$

$\partial_{\vartheta}\left\{1+i \eta\left(\omega-\omega_{* e}\right)^{-1} \vartheta^{2}\right\}^{-1} \partial_{\vartheta}\left\{\left(1+i \eta \omega^{-1} \vartheta^{2}\right) n_{e}-\left(\omega_{* e} / \omega\right) \Phi\right\}=0$

Integration of Eqs. (16) and (17) yields

$n_{e}-\Phi=B_{1}+B_{0}\left\{i \eta\left(\omega-\omega_{* e}\right)^{-1} \vartheta-\vartheta-1\right\}$

Asymptotic matching to Eq. (15) determines the integration constants. Therefore, the large- $\vartheta$ asymptotic behavior of Eq. (18) is

$\mathrm{n}_{\mathrm{e}}-\Phi \approx\left(\omega_{* \mathrm{e}} / \omega-1\right)\left\{1+\mathrm{i} \mathrm{r}_{0} \Delta^{\prime} \eta \vartheta / 2 \pi^{2} \mathrm{NqS}\left(\omega-\omega_{* \mathrm{e}}\right)\right\}$

Finally, we consider the inertial layer (deeply resistive region) where $\eta \vartheta^{2}>\omega_{* e}$ is satisfied. In this region, the forms of magnetic field line bending term in Eq. (11) and the parallel compression (sound wave) term in Eq. (12) can be simplified because the disssipation dominates reactive effects. Then, Eqs. (11) and (12) become, 
$\left(\mathrm{V}_{\mathrm{A}} / \mathrm{qR}\right)^{2} \partial_{\vartheta}^{2}\left(\mathrm{n}_{\mathrm{e}}-\Phi\right)-i \eta \operatorname{\omega o\vartheta }^{2} \Phi=0$

and

$\left(C_{S} / q R\right)^{2} \partial_{\vartheta}^{2} n_{e}$

$=-\left\{1+i\left(\gamma_{v}+\beta_{n}\right) / \omega\right\} \omega^{2}\left\{\left(1-i \beta_{n} / \omega\right) n_{e}-\left(\omega_{*} e^{/ \omega-b_{\vartheta} S^{2} \vartheta}\right) \Phi\right\}$.

The difference in inertial layer dynamics distinguishes the semi-collisional drift-tearing modes from the strongly collisional drift-tearing modes. In the semi-collisional regime, the currrent channel width $\Delta_{D}=L_{S}\left(\omega_{*} v_{e i}\right)^{1 / 2} / k_{\vartheta} v_{T e}$ is narrower than the ion Larmor radius size (at the electron temperature) $\rho_{S}$, and the induction field $\omega \Psi$ accounts for most of $E_{\|}$inside $\Delta_{D}$. Therefore, the inertial layer dynamics is almost purely electromagnetic. ${ }^{5}$ On the contrary, in the strongly collisional regime, $\rho_{S}>\Delta_{D}$ is satisfied and the electrostatic component of the parallel electric field is significant. We consider the strongly collisional drift-tearing modes first.

\section{A. Strongly Collisional Regime:}

Density evolution in the inertial layer is governed by the $\mathbf{E} \times \mathbf{B}$ convection and the ionization source. Therefore, Eq. (21) simplifies to

$\left(1-\mathrm{i} \beta_{\mathrm{n}} / \omega\right) \mathrm{n}_{\mathrm{e}}=\left(\omega * \mathrm{e}^{/ \omega) \Phi}\right.$

Then, Eq. (20) becomes a Weber equation for $\Phi$ with a solution which decays for large $\vartheta$ and $\operatorname{Im} \omega>0$, 
$\Phi=\mathrm{y}^{1 / 2} \mathrm{~K}_{1 / 4}\left(\mathrm{y}^{2} / 2\right)$,

where $y=c_{Y} \vartheta, c_{Y}=\left[i \eta\left(V_{A} / q R\right)^{-2} \omega\left(\omega-i \beta_{n}\right)\left(\omega_{*} e^{-\omega+i} \beta_{n}\right)^{-1}\right]^{1 / 4}$

and $K_{1 / 4}$ is the modified Bessel function. Finally, matching the small- $\vartheta$ asymptotic behavior of Eq. (23) to Eq. (19), we obtain

$\left(\omega-\omega_{* e}\right)^{4}\left(\omega-i \beta_{n}\right) \omega=i \gamma_{F K R}{ }^{5}\left(\omega-\omega_{* e}-i \beta_{n}\right)$,

where $\gamma_{\mathrm{FKR}}=\left(\Gamma(1 / 4) \mathrm{r}_{0} \Delta^{\prime} / 4 \pi^{2} \Gamma(3 / 4) \mathrm{NqS}\right)^{4 / 5} \eta^{3 / 5}\left(\mathrm{~V}_{\mathrm{A}} / \mathrm{qR}\right)^{-2 / 5}$

When $\beta_{n}$ is ignorable, Eq. (24) reduces to the results of Ref. 5. We also note that the ionization effect is relevant only in the drift-tearing limit. If $\omega \gg \omega_{* e}$, we recover the classical tearing mode dispersion relation of Furth, Killeen, and Rosenbluth. ${ }^{3}$ Low-N linear tearing modes in the present day tokamak edge are mostly in the drift tearing regime, where $\omega$ $\cong \omega_{*_{e}} \gg \gamma$. First, treating $\beta_{n}$ term perturbatively, we obtain

$\left.\omega \equiv \omega_{* e}+i\left(\gamma_{F K R} 5 / \omega_{*}\right)^{2}\right)^{1 / 3}\left\{1-i \beta_{n}\left(\omega-\omega_{* e}\right)^{-1}\right\}^{1 / 3}$

$\left.\equiv \omega_{* e}+i\left(\gamma_{F K R} 5 / \omega_{*} e^{2}\right)^{1 / 3}\left\{1-\mathrm{i}^{2 / 3} \beta_{\mathrm{n}}\left(\gamma_{\mathrm{FKR}} 5 / \omega_{* e}\right)^{2}\right)^{-1 / 3}\right\}^{1 / 3}$

which indicates that $\beta_{\mathrm{n}}$ reduces the growth rate of the strongly collisional drift-tearing mode. This perturbative treatment requires $\gamma_{\mathrm{FKR}} 5 / 3 \gg \omega_{*} \mathrm{e}^{2 / 3} \beta_{\mathrm{n}}$ as well as $\omega_{* \mathrm{e}} \gg \gamma_{\mathrm{FKR}} \gg \beta_{\mathrm{n}}$. Simple inspection of Eq. (24) shows that the drift-tearing mode is completely stabilized for $\Delta^{\prime}$ $<\Delta^{C 1}$, where $\Delta^{C 1}$ is given below. Writing $\omega=\omega_{*} \mathrm{e}^{+\delta \omega}$ and separating the real and imaginary parts of Eq. (24), we obtain at the marginal stability, 


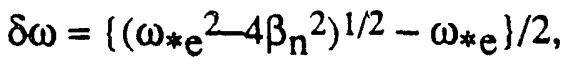

and

$\delta \omega^{2} \beta_{\mathrm{n}}{ }^{3}=\gamma_{\mathrm{FKR}}{ }^{5}$.

From the definition of $\gamma_{F K R}$ we obtain the linear threshold value of $\Delta^{\prime}$,

$\mathrm{r}_{0} \Delta^{C 1}=\left(4 \pi^{2} \Gamma(3 / 4) N q S / \Gamma(1 / 4)\right)\left(\beta_{n} / \eta\right)^{3 / 4}\left(\delta \omega q R / V_{A}\right)^{1 / 2}$

We note that this threshold value is extremely small since $\delta \omega \equiv-\beta_{n}{ }^{2 / \omega_{* e}}$. For typical present day tokamak edge parameters, the sound wave effects produce a larger, therefore, more relevant value of $\Delta^{\prime}$ threshold. We derive that threshold in the presence of ionization and charge exchange in the next section. In summary, the ionization has a stabilizing influence on the strongly collisional drift-tearing modes in the absence of sound wave dynamics. The physical reason is as follows. In the absence of sound wave dynamics, we can rearrange Eq. (8), using Eqs. (6) and (7).

$$
\begin{aligned}
& -\left\{\eta_{s}+i\left(\rho_{s} v_{A} k_{\| 1}\right)^{2} /\left(\omega-i \beta_{n}\right)\right\} \nabla_{\perp} 2 \Psi \\
& =\left(1-\omega_{*} \mathrm{e} / \omega\right) \mathrm{E}_{\|}+\mathrm{i}\left\{\left(\omega_{* \mathrm{e}} / \omega\right)\left(1-\mathrm{i} \beta_{\mathrm{n}} / \omega\right)^{-1}-\left(\omega_{* \mathrm{e}} / \omega\right)\right\} \mathrm{k}_{\|} \Phi .
\end{aligned}
$$

In the strongly collisional regime, the radial dependence of the effective conductivity (the second term on the left hand side) is negligible, and the mode width in radial direction is determined by the condition $E_{\| \rightarrow} \rightarrow 0$. Therefore, $\Phi$ is not ignorable throughout the mode width. If $\beta_{n} \rightarrow 0$, the right hand side which characterizes the driving force to induce parallel perturbed current is proportional to $\left(1-\omega_{* e} / \omega\right) \mathrm{E}_{\|}$. Integration of the parallel current in radial direction 
followed by matching to $\Delta^{\prime}$, shows that $\omega \equiv \omega_{* e}$ must be satisfied. ${ }^{7}$ Now finite $\beta_{\mathrm{n}}$ breaks up the near cancellation of the perturbed electric field and the pressure gradient along the magnetic field, and gives stabilizing influence.

\section{B. Semi-collisional Regime:}

In this regime, $\rho_{S}<\Delta_{D}$ is satisfied and the electrostatic effects can be ignored inside the inertial layer. $n_{e} \gg \Phi$ leads Eqs. (20) and (21) to

$\left(\mathrm{V}_{\mathrm{A}} / \mathrm{qR}\right)^{2} \partial_{\vartheta}^{2} \mathrm{n}_{\mathrm{e}}-\mathrm{i \eta} \omega \vartheta^{2} \Phi=0$

and

$\left(1-i \beta_{n} / \omega\right) n_{e}+b_{\vartheta} S^{2} \vartheta^{2} \Phi=0$

The well-behaved solution of Eqs. (30) and (31) is

$n_{e}=\exp (-\alpha \vartheta)$

where $\alpha=\left[-i \eta\left(\omega-i \beta_{n}\right) / b_{\vartheta}\right]^{1 / 2}\left(S V_{A} / q R\right)^{-1}$

By matching the small- $\vartheta$ asymptotic behavior of Eq. (32) to Eq. (19), we obtain the dispersion relation for the semi-collisional drift-tearing mode, 
$\left(\omega-\omega_{*}\right)\left(\omega-i \beta_{n}\right)^{1 / 2}=i^{3 / 4}\left(\rho_{s} \Delta^{\prime} / 2 \pi^{2}\right) \eta^{1 / 2} V_{A} / q R$

This again reduces to the results of Ref. 5 when $\beta_{n}$ is ignorable. In the drift-ter ring regime, $\omega$ $\equiv \omega_{* \mathrm{e}} \gg \gamma$, and the growth rate is given by

$\gamma=\left(1-\beta_{\mathrm{n}} / 2 \omega_{* \mathrm{e}}\right)\left(\rho_{\mathrm{S}} \Delta^{\prime} / 2^{3 / 2} \pi^{2}\right) \eta^{1 / 2} \mathrm{~V}_{\mathrm{A}} / \omega_{*} \mathrm{e}^{1 / 2} \mathrm{qR}$

This reduction of growth rate is relatively smaller than the reduction in the strongly collisional regime. On the contrary to the strongly collisional regime, the radial mode width of the semi-collisional drift-tearing mode is set by the radial dependence of the effective parallel conductivity; i.e., the inverse of the coefficient of $\nabla_{\perp} 2 \Psi$ on the left hand side of Eq. (29). Within this current channel, small $k_{\|}$makes the electrostatic contribution to the parallel electric field ignorable. Therefore, the aforementioned ionization modification of driving force does not cause a significant change in the growth rate. Instead, the reduction of growth rate comes from the $\beta_{\mathrm{n}}$-dependence of the current channel width.

\section{Atomic Physics Effects on Ion Sound Wave Induced Tearing Instability Threshold}

In this section, we investigate the effects of ionization and charge exchange on the ion sound wave induced tearing instability threshold. We note that the ion sound wave effects on drift tearing modes have been studied by Bussac et al. ${ }^{17}$ numerically in the strongly collisional regime and both analytically and numerically in the semi-collisional regime. Also extension to toroidal geometry has been performed analytically. 19 We recall that the ion sound wave 
dynamics has been ignored in the previous section by simplified treatments of the inertial layer physics described by Eqs. (20) and (21). Here, the ion sound wave dynamics is included perturbatively.

\section{A. Strongly Collisiona! Regime:}

The zero-th order soiution of the previous section satisfies Eq. (22); i.e., $\left(1-i \beta_{n} / \omega\right) n_{e} e^{0}=$ $\left(\omega_{* \mathrm{e}} / \omega\right) \Phi 0$. Then, by including the first term of Eq. (21) perturbatively, we obtain from Eqs. (20) and (21),

$$
\left(V_{A} / q R\right)^{2} \partial_{\vartheta}^{2}\left(n_{e}^{1-\Phi^{1}}\right)-i \eta \omega \vartheta^{2} \Phi^{1}=0
$$

and

$\left(\mathrm{C}_{\mathrm{g}} / \mathrm{qR}\right)^{2} \mathrm{a}_{\vartheta}{ }^{2} \mathrm{n}_{\mathrm{e}}^{\mathrm{o}}$

$=-\left\{1+i\left(\gamma_{v}+\beta_{n}\right) / \omega\right\} \omega^{2}\left\{\left(1-i \beta_{n} / \omega\right) n_{e}{ }^{1}-\left(\omega * e / \omega-b_{\vartheta} S^{2} \vartheta 2\right) \Phi^{1}\right\}$.

Elimination of $\mathrm{n}_{\mathrm{e}}^{1}$ yields

$$
\left(r Y^{2}-y^{2}\right) \Phi=c_{B} \partial Y^{2}\left(y^{2} \Phi 0\right)
$$

where $c_{B}=\left(C_{S} / V_{A}\right)^{2}(i \eta)^{1 / 2}\left(V_{A} / \omega q R\right)\left(\omega_{*} / \omega\right)\left(1-i \beta_{n} / \omega\right)^{-1 / 2}\left\{1+i\left(\gamma_{v}+\beta_{n}\right) / \omega\right\}^{-1}$

$\left(\omega_{*} e^{-\omega+i \beta_{n}}\right)^{-3 / 2}$. The well-behaved solution of Eq. (37) is given by

$$
\Phi^{1}=-y^{1 / 2} I_{1 / 4}\left(y^{2} / 2\right) \int_{y} d t t^{1 / 2} K_{1 / 4}\left(t^{2} / 2\right) c_{B} \partial_{t}^{2}\left(t^{2} \Phi^{0}\right)
$$




$$
-y^{1 / 2} K_{1 / 4}\left(y^{2} / 2\right) \int y d t t^{1 / 2} I_{1 / 4}\left(t^{2} / 2\right) c_{B} \partial_{t}^{2}\left(t^{2} \Phi^{0}\right)
$$

with $\Phi 0$ given in Eq. (23).

The small-y asymptotic behavior is given by

$\Phi^{1} \cong-3 \pi 2^{-9 / 2} \Gamma(3 / 4) c_{B} y$.

Combining $\Phi^{1}$ and $n_{e}^{1}$ to the zero-th order solution, we obtain the small- $\vartheta$ asymptotic behavior of $n_{e}-\Phi$.

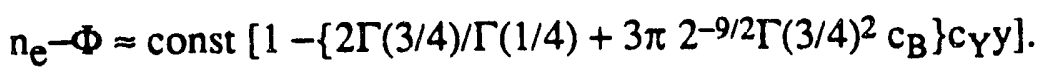

Matching Eq. (40) to Eq. (19), we obtain

i $r_{0} \Delta^{\prime} \eta / 2 \pi^{2} \mathrm{NqS}\left(\omega-\omega_{* e}\right)=\left[i \eta\left(V_{A} / q R\right)^{-2} \omega\left(\omega-i \beta_{n}\right)\left(\omega_{* e} e^{-\omega+i} \beta_{n}\right)^{-1}\right]^{1 / 4}$ $\left\{2 \Gamma(3 / 4) / \Gamma(1 / 4)+3 \pi 2^{-9 / 2} \Gamma(3 / 4)^{2} c_{B}\right\}$.

Here, the last term inside the parenthesis on the right hand side is the contribution from the ion sound wave dynamics. The threshold for marginal stability is obtained by balancing this term against the left hand side of Eq. (40).

$$
\begin{aligned}
& r_{0} \Delta^{\prime}=i^{1 / 4} 3 \pi^{22-7 / 2} \Gamma(3 / 4)^{2} \mathrm{NqS}\left(C_{S} / V_{A}\right)^{2}\left(V_{A} / \omega q R\right)^{1 / 2}(\omega / \eta)^{1 / 4}\left(\omega_{*} / \omega\right)\left(1-i \beta_{n} / \omega\right)^{-1 / 4} \\
& \left.\left\{1+i\left(\gamma_{v}+\beta_{n}\right) / \omega\right\}^{-1}\left(1-\omega_{* e} / \omega\right)\left\{1-\left(\omega_{* e}+i \beta_{n}\right) / \omega\right)\right\}^{-7 / 4}
\end{aligned}
$$

In Eq. (42), the last two factors depend on $\Delta^{\prime}$ through the dispersion relation given by Eq. 
(24). When $\gamma_{v}, \beta_{n} \rightarrow 0$, we obtain

$r_{0} \Delta_{B C}=\left\{3 \pi^{4} 2^{-3 / 2} \Gamma^{\prime}(3 / 4)^{3 / \Gamma} \Gamma(1 / 4)\right\}^{1 / 2} \mathrm{NqS}\left(\mathrm{C}_{\mathrm{g}} / \mathrm{V}_{\mathrm{A}}\right)\left(\omega_{*} / \eta\right)^{1 / 4}$

This analytical formiala confirms the previous result in Ref.17. There, dimensional analysis has been used for scalings and the coefficient has been numerically computed. The right hand side scales like $T_{e} e^{7 / 4 / B_{t}}{ }^{3 / 2}$ for fixed $q$ and $S$. It has been reported in Ref. 20 that these scalings compare favorably with $\mathrm{L} \rightarrow \mathrm{H}$ transition data 2 from DIII-D if the stabilization of the drift tearing mode is related to the transition.

Now, in the presence of atomic physics effects, Eq. (42) with Eq. (25) yields,

$\Delta^{C 2}=\left(1+\beta_{n} \omega_{*} e^{2 / 3 / 2} \gamma_{F K R}{ }^{5 / 3}\right) \Delta_{B C}$,

where $\gamma_{F K R}$ is evaluated at $\Delta^{\prime}=\Delta_{B}$, and $\omega_{* e} \gg \gamma_{F K R} \gg \beta_{n}, \gamma_{v}$ has been assumed. This is in qualitative agreement with the experimental trend observed in TFTR that the gas puff makes super shot to $H$-mode transition easier. ${ }^{21}$ This threshold $\Delta \Delta^{C 2}$ which increases with $\beta_{n}$ is typically much larger than $\Delta^{\mathrm{C} 1}$ in Eq. (28), and thus, more relevant.

\section{B. Semi-Collisional Regime:}

In the semi-collisional regime, the ion sound wave stabilization effects come from the deviation of the electron density response from the Boltzmann relation. ${ }^{19}$ If the electron density response is Boltzmann, Eq. (21) becomes

$\partial_{\vartheta}^{2} \Phi=\left\{\delta k^{2}+k^{4} \vartheta^{2} / 4\right) \Phi$, 
where $K=(-i \omega)^{1 / 2}\left[4 b_{\vartheta} S^{2} q^{2} R^{2}\left\{1+i\left(\gamma_{v}+\beta_{n}\right) / \omega\right\} / C_{s}^{2}\right]^{1 / 4}$, and $\delta=-i\left(\omega \mathrm{q} R / C_{S}\right)\left\{1+i\left(\gamma_{v}+\beta_{n}\right) / \omega\right\}^{1 / 2}\left\{1-\left(\omega * e^{+i} \beta_{n}\right) / \omega\right\} b_{\vartheta}{ }^{-1 / 2} S^{-1}$. The solution is given by a Parabolic Cylinder function, 22

$\Phi=\mathrm{U}(\delta, \kappa \vartheta)$

Now, the deviation from the Boltzmann relation $\mathrm{n}^{\mathrm{N}}=\mathrm{n}_{\mathrm{e}}-\Phi$ can be perturbatively calculated from Eq. (20),

$\left(V_{A} / q R\right)^{2} \partial_{\vartheta}^{2} n^{N}-i \eta \omega \vartheta^{2} U(\delta, \kappa \vartheta)=0$,

integrating twice, we obtain

${ }_{n} N=i \eta \omega\left(V_{A} / q R\right)^{-2} \int_{\vartheta} d t t^{2}(t-\vartheta) U(\delta, \kappa \vartheta)$

Adding $\mathrm{n}^{\mathrm{N}}$ to Eq. (31) and matching the small- $\vartheta$ asymptotic behavior to Eq. (16), we obtain the following dispersion relation which includes the sound wave contribution alongside the atomic physics effects. We note that the Boltzmann response does not contribute to the asymptotic matching.

$r_{0} \Delta^{\prime} / 2 \pi^{2} \mathrm{NqS}\left(\omega-\omega_{* e}\right)=\left[\eta\left(\omega-i \beta_{n}\right) / b_{\vartheta}\right]^{1 / 2} q R / S V_{A}-i \eta \omega\left(V_{A} / q R\right)^{-2}\left(\omega-\omega_{* e}\right)^{-1} I_{2}$,

where $I_{2}=\int_{0} d t t^{2} U(\delta, \kappa t) / U(\delta, 0)$. Thus, the threshold of the semi-collisional drift-tearing mode is given by 
$r_{0} \Delta_{S C}=-2 \pi^{2} N q S\left(V_{A} / q R\right)^{-2} \omega_{*} e^{2} I_{2}$

For $\delta \rightarrow 0$, then Eq. (49) becomes

$r_{0} \Delta C 3=\left\{\pi^{2} \Gamma(3 / 4) / 2 \Gamma(1 / 4)\right\}\left\{1-3\left(\gamma_{v}+\beta_{n}\right) / \omega_{*}\right\}\left(q R / S L_{n}\right)^{1 / 2}\left(C_{S} / V_{A}\right)^{2} r_{0} / \rho_{S}$.

In the absence of atomic physics effects, we recover the result of Ref. 17. This stabilizing effect persists in a toroidal geometry with good average curvature. Eq. (50) scales like $\mathrm{T}_{\mathrm{e}} / \mathrm{B}_{\mathrm{t}}{ }^{2}$. Both $\gamma_{\mathrm{v}}$ and $\beta_{\mathrm{n}}$ lower the threshold. since both the ionization and charge exchange exert drag on the ion motion along the magnetic field line, the stabilizing influence of ion sound wave propagation becomes less effective. In the other asymptotic limit ( $\delta \gg 1$ ), Eq. (49) becomes

$\mathrm{r}_{\mathrm{o}} \Delta^{\mathrm{C} 4}=2^{1 / 2}\left(\omega_{* \mathrm{e}} / \beta_{\mathrm{n}}\right)^{3 / 2}\left(\mathrm{C}_{\mathrm{s}} / \omega_{* \mathrm{e}} \mathrm{qR}\right)^{3 / 2}$

Thus, the decrease of $\Delta^{C}$ continues as $\beta_{n}$ is increased to a larger value exceeding $b_{\vartheta}{ }^{1 / 2} S C_{S} / q R$.

\section{Summary}

The effects of sound wave dynamics, ionization, and charge exchange on the drift-tearing modes are investigated in this paper. The drift-tearing mode dispersion relations are derived analytically for both strongly collisional regime and semi-collisional regime. In the strongly collisional regime, ionization reduces the effective force to drive the parallel current by 
the following formula,

$F_{e f f}=\left(1-\omega_{* e} / \omega\right) E_{\|}+i\left(\left(\omega * e^{\left./ \omega)\left(1-i \beta_{n} / \omega\right)^{-1}-(\omega * e / \omega)\right\} k_{\|} \Phi}\right.\right.$

Consequently, it raises the instability threshold $\Delta_{\mathrm{BC}}$ due to sound wave by,

${ }_{\Delta} C 2=\left(1+\beta_{n} \omega_{*} e^{2 / 3 / 2} \gamma_{F K R}{ }^{5 / 3}\right) \Delta_{B C}$.

This stabilizing influence persists when this threshold is exceeded, and the linear growth rate is reduced by the same order as shown in Eq. (25). The effect of charge exchange is small.

In the semi-collisional regime, the radial mode width of the drift-tearing mode is set by the radially dependent effective parallel conductivity. Within this current channnel, small $k_{\|}$ makes the electrostatic contribution to the parallel electric field ignorable and the mode character is predominantly electromagnetic. Therefore, the aforementioned ionization modification of driving force does not cause a significant change in the growth rate. Instead, the reduction of growth rate comes from the $\beta_{n}$-dependence of the current channel width as shown in Eq. (34). Both the ionization and charge exchange exert drag on the ion motion along the magnetic field line, and the stabilizing influence of ion sound wave propagation becomes less effective. The instability threshold $\Delta_{\mathrm{BS}}$ due to sound wave is reduced by,

$\Delta^{C 3}=\left(1-3\left(\gamma_{v}+\beta_{n}\right) / \omega_{* e}\right) \Delta_{B S}$. This trend of the threshold reduction continues as $\beta_{n}$ is increased to a larger value exceeding $b_{\vartheta} 1 / 2 S C_{\mathcal{S}} / q R$ as shown in Eq. (51). 


\section{Acknowledgments}

The author would like to thank G. G. Craddock, C. E. Bush, and M. Zarnstorff for useful discussions.

This work was supported by U.S. Department of Energy Contract No. DE-AC02-76-CHO-3073. 


\section{References}

1 K. Toi, J. Gernhardt, O. Kluber, and M. Kornherr, Phys. Rev. Lett. 62, 430 (1989).

2 K. H. Burrell, T. N. Carlstrom, E. J. Doyle, R. J. Groebner, T. Lehecka, N. C. Luhman, Jr., H. Matsumoto, T. H. Osborne, W. A. Peebles, and R. Philipona, Phys. Fluids B 2, 1405 (1990).

3 H. P. Furth, J. Killeen, and M. N. Rosenbluth, Phys. Fluids 6, 459 (1963).

4 B. Coppi, Phys. Fluids 7, 1501 (1964).

5 J. F. Drake and Y. C. Lee, Phys. Fluids 20, 1341 (1977).

6 C. S. Chang, R. R. Dominguez, and R. D. Hazeltine, Phys. Fluids 24, 1655 (1981).

7 S. C. Cowley, R. M. Kulsrud, and T. S. Hahm, Phys. Fluids 29, 3230 (1986).

8 J. D. Callen and K. C. Shaing, Phys. Fluids 28, 1845 (1985).

9 J. W. Connor and L. Chen, Phys. Fluids 28, 2001 (1985).

10 T. S. Hahm, Phys. Fluids 31, 3709 (1988).

11 R. Fitzpatrick, Phys. Fluids B 2, 2636 (1990).

12 T. S. Hahm and P. H. Diamond, Phys. Fluids 30, 133 (1987).

13 K. Toi, et al., Phys. Rev. Lett. 64, 1895 (1990).

14 A. S. Ware, P. H. Diamond, B. A. Carreras, J. N. Leboeuf, and D. K. Lee, Phys. Fluids B 4, 102 (1992).

15 Y. Z. Zhang and S. M. Mahajan, Phys. Fluids B 4, 207 (1992).

16 M. A. Beer and T. S. Hahm, Phys. Fluids B 4, 2567 (1992).

17 M. N. Bussac, D. Edery, R. Pellat, and J. L. Soule, Phys. Rev. Lett. 40, 1500 (1978).

18 G. G. Craddock and P. W. Terry, B 3, 3286 (1991).

19 T. S. Hahm and Liu Chen, Phys. Fluids 29, 1891 (1986).

20 H. R. Strauss, Phys. Fluids B 4, 934 (1992). 
21 C. E. Bush, Private Communications (1992).

22 M. Abramowitz and I. A. Stegun, Handbook of Mathematical Functions (Dover, New York, 1972). 
Dr. F. Peotoni, Univ. of Wollongong, AUSTRULIA

Prof. M.H. Brennan, Univ. of Sydney, AUSTRALIA

Plasma Posearch Lub., Australian NaL. Univ., AUSTRALIA

Prof. I.A. Jones, Flinders Univ. AUSTRULLIA

Prot. F. Cap, Inst for Theoretical Physics, AUSTRIA

Prof. M. Heinder, Instiul for Theoretische Physik, AUSTRIA

Prof. M. Goossens, Astronomisch InstituUt, BELGIUM

Ecole Royede Mitivin, Lob. do Phy. Plaemas, BELGIUM

Commission-Europosn, DG. XII-Fusion Proo., BELGIUM

Prof. R. Bouciqut, Rijkeuniwercitit Gent, BELGIUM

Dr. P.H. Sakanaka, Instituto Firica, BRUZIL

Instituto Naciond De Prequises Espacieie-INPE, BRAZIL

Documents Orico, Alomic Eneroy of Ceneda LAd., CANADA

Dr. M.P. Bectynnakj, MPB Technologies, Inc., CANADA

Dr. H.M. Skaregard, Univ. of Sackalchewen, CANADA

Prof. J. Toichmam, Univ. of Montreal, CANADA

Prof. S.R. Sroeniveen, Univ. of Colgery, CANADA

Prof. T.W. Johneton, INAS-Energio, CANADA

Dr. R. Boton, Contre canedion de husion magnátique, CANADA

Dr. G.R. Janes, Univ. of Nborti, CANADA

Dr. P. Lukstc, Komenekcho Iniverszita, CZECHOSLOVAKIA

The Librarian, Cultem Leworalory. ENGLAND

Librery, R61. Ruthertord Apploton Let story. ENGLAND

Mrs. S.A. Hulchineon, JET Librery, ENGLAND

Dr. S.C. Sherma, Univ. of South Pecific, FWI ISLANDS

P. Methonen, Univ. of Haleinki, FINLAND

Prot. M.N. Buseac, Ecoto Polychnique., FRANCE

C. Nourbt, Lob. Physique des Mitioux loniads, FRANCE

J. Radol, CENCADARACHE - Bat 506, FRANCE

Prot. E. Economou, Univ. of Creto, GREECE

Ms. C. Rinni, Univ. of loannina, GREECE

Dr. T. Muel, Acadomy Bibliographic Ser., HONG KONG

Preprint Librey, Hungarian Academy of Sci., HUNGARY

Dr. B. DesGupta, Sana inst of Nuctoer Physics, INDIA

Dr. P. Kaw, Inst. for Plasma Reseerch, INDIA

Dr. P. Rowenew, lereel inat of Technology, ISPAEL

Librerien, Intemationed Conter for Theo Physics, ITALY

Miss C. De Palo, Associazione EURATOM-ENEA, ITALY

Dr. G. Groseo, lestubo d Fieica del Plasma. ITALY

Prot. G. Postengni, letious Gas boniezedi Det Cnr, ITALY

Dr. H. Yemato, Toshiba Res a Del Center, JAPAN
Prof. I. Kewakemi, Hiroshima Univ., JAPAN

Prof. K. Nishikawa, Hiroshima Univ., JAPAN

Director, Jeaen Atomic Energy Research Inst, JÄPAN

Prof. S. Itoh, Kyushu Univ., JAPAN

Research Into. Ctr., National Instit. tor Fusion Science, JAPAN

Prot. S. Tenaka, Kyoto Univ., JAPAN

Librey, Kyolo Univ., JAPAN

Prot. N. Inowe, Univ. of Tokyo, LAPAN

Socretary, Plaema Section, Eloctrotechnical Lab., JAPAN

S. Mori, Technical Advicor, LAERI, LAPAN

Dr. O. Mireni, Kumanoto inst of Technology, JAPAN

J. Hyoon-Sook, Korea Alomic Enorgy Rescarch Inst, KOREA

O.I. Chai, The Korea Adv. Inst of Sai. Tech., KOREA

Prof. 8.S. Liby, Univ. of Weikab, NEW ZEALAND

Inct of Phyrics. Chinese Aced Sci PEOPLE'S REP. OF CHINA

Library, Inst of Ptasma Phycias, PEOPLE'S REP. OF CHINA

Tringhua Univ. Library. PEOPLE'S REPUBLIC OF CHINA

Z. Li, S.W. Inat Phyrics, PEOPLE'S REPUBUC OF CHINA

Prof. J.A.C. Cabred, Instituto Superior Tecnico, PORTUGAL

Dr. O. Porus, Al I CUzA Univ., ROMaNIA

Dr. J. do Villiers, Fueion Studios, AEC, S. AFRICA

Prof. M.A. Hellberg, Univ. of Matal, S. AFRICA

Prot. D.E. Km, Pohang inst. of Sai. I Tech., SO. KOPEA

Prot. C.I.E.M.A.T, Fuaion Diviaion Library, SPAIN

Dr. L Stormo, Univ. Of UMEA, SWEDEN

Librery, Royed inat. of Technology, SWEDEN

Prol. H. Withelmeon, Chalmers Univ. of Tech., SWEDEN

Centre Phys. Das Plasmas, Ecole Polytech, SWITEERLAND

Bibliothoek, Inst. Voor Plasma-Fycica, THE NETHERLANDS

Asst Prot. Dr. S. Cakir, Midde East Tech. Univ., TURKEY

Dr. V.A. Gulthikh,Sai. Rese. Inat Eloctrophys.I Apparatus, USSR

Dr. O.D. Aputov, Siberian Brench of Academy of Sa., USSR

Dr. G.A. Eliecer, I.V. Kurchatov Inst, USSA

Libresian. The Ukr.SSR Acedemy of Scioncos, USSR

Dr. LM Kovizhnykh, inst. of Generd Physics, USSA

Kerntorechungsanlege GmbH, Zentrabibiothek, W. GERMANY

Bibliothak, Inse. For Plasmalorechung, W. GERMANY

Prot. K. Schindler, Ruhr-Univereidt Bochum, W. GERMANY

Dr. F. Wegner, (ASOEX), Max-Planck-Inotitut, W. GERMANY

Librerien, Max-Plenck-Institut, W. GERMANY

Prot. R.K Janow, Inst of Ptyrics, YUCOSLAVIA 

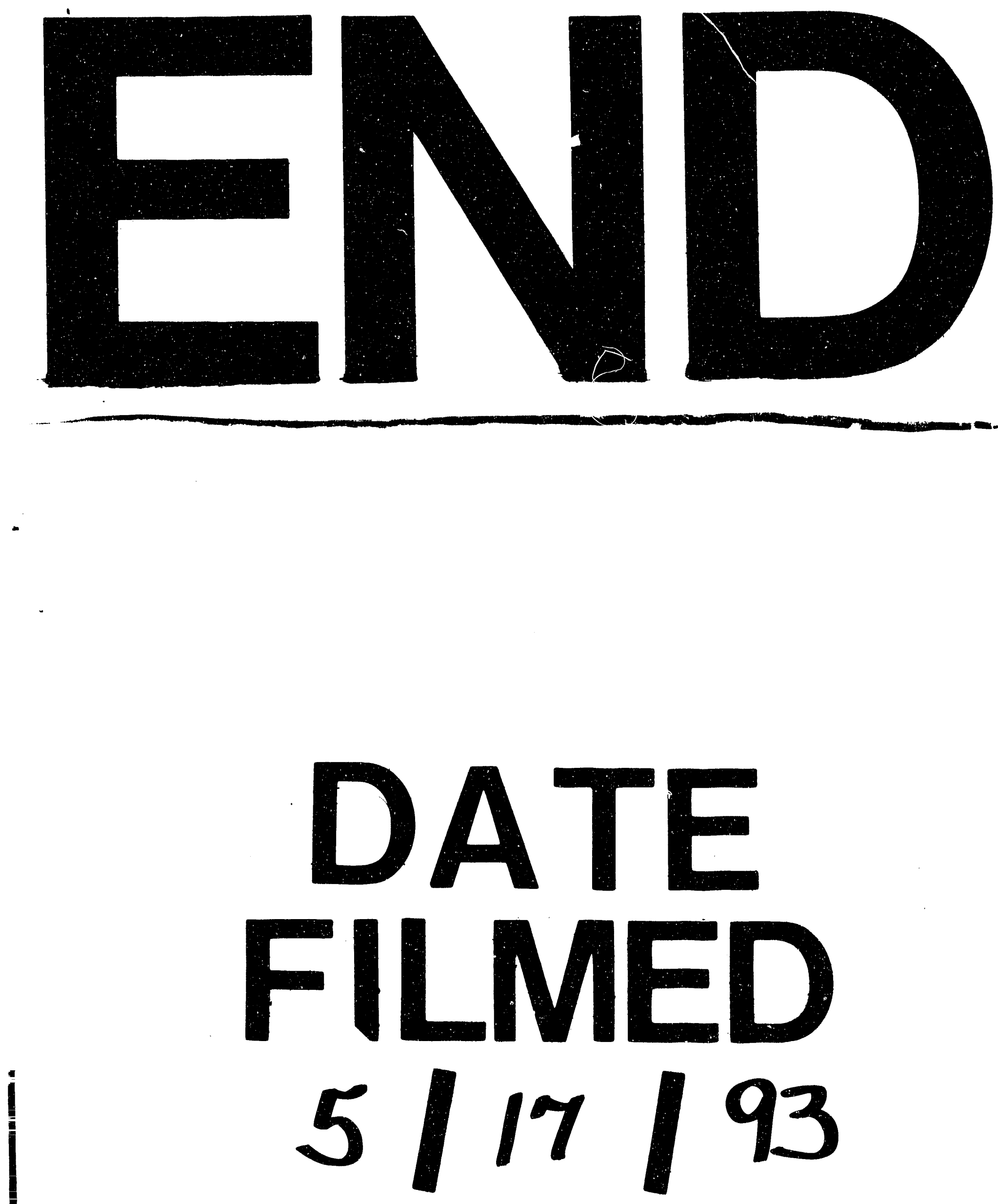
